\title{
Interaction of vacancies with a grain boundary in aluminum: A first-principles study
}

\author{
Gang Lu and Nicholas Kioussis \\ Department of Physics, California State University Northridge, Northridge, California 91330-8268
}

(Received 24 January 2001; published 18 June 2001)

\begin{abstract}
We present a theoretical study of the interaction of vacancies with a tilt grain boundary in aluminum based on the density functional theory. The grain boundary volume expansion and vacancy induced contraction are calculated and compared for the nearest-neighbor atoms, and the former is found to be smaller than the latter. The formation energy of a vacancy placed at various layers in the grain boundary has been calculated and we find that it costs more energy to form a vacancy at the boundary plane than in bulk, although the rest of the grain boundary region does attract vacancies. The microscopic mechanisms of grain boundary sliding and migration are investigated thoroughly with and without a vacancy. We find that although the vacancy can hinder the grain boundary motion by tripling the energy barrier of sliding and migration, it cannot inhibit or even delay the migration process. The vacancy placed at the first layer from the interface is found to be trapped at the layer and not able to follow the migrating interface.
\end{abstract}

DOI: 10.1103/PhysRevB.64.024101

PACS number(s): 61.72.Bb, 61.72.Ji, 61.72.Mm

\section{INTRODUCTION}

Vacancies are the simplest lattice defects and play an important role for various properties of materials, such as kinetic, thermodynamic, electrical, optical and even mechanical properties, to name a few. Similarly, grain boundaries as planar defects, are also responsible for the various material properties. ${ }^{1,2}$ While there have been extensive theoretical studies aimed at either vacancies or grain boundaries individually, much less effort has been devoted to the understanding of the collective behavior of grain boundaries and vacancies, especially from parameter-free $a b$ initio studies. For example, ab initio calculations have been used with great success to study the vacancy formation energy and the vacancy-induced relaxation in different metals, ${ }^{3,4}$ the vacancy diffusion energy barrier and the annihilation process in semiconductors, ${ }^{5-7}$ etc. However, all these calculations are confined to either bulk systems or surfaces. On the other hand, grain boundaries (pure or with impurities) have attracted a great deal of attention recently. For example, $a b$ initio calculations have been performed for the grain boundary energies and the atomic structures of the pure $\Sigma 11$ tilt and $\Sigma 3$ twin grain boundaries in aluminum, ${ }^{8}$ and the effect of impurity on the grain boundary cohesion in $\mathrm{Ni}_{3} \mathrm{Al}^{9}$ However, these studies do not involve vacancies in the grain boundaries and examine only the properties of the grain boundaries themselves. Therefore, one of the goals in this paper is to investigate the combined effect of grain boundaries and vacancies in aluminum using $a b$ initio calculations and to explore the possible interactions between the point and planar defects.

While the equilibrium structures of grain boundaries have been extensively studied, only very limited research has focused on the atomistic simulation of grain boundary sliding and migration. ${ }^{10,11,13,14}$ The former process involves the relative displacement of the two constituent grains in a direction parallel to the boundary interface, while the latter involves the motion of the interface in a direction perpendicular to the boundary plane. Grain boundary sliding/migration is considered to be one of the principal mechanisms of plastic defor- mation of polycrystalline materials at intermediate-to-high temperatures (above $0.6 T_{m}$, where $T_{m}$ is the melting point) and it contributes to creep deformation and intergranular fracture, which can in turn be significantly affected by the presence of vacancies. ${ }^{2,15}$ These two modes of grain boundary motion can be correlated from a simple geometrical analysis. ${ }^{16}$ Very recently, Molteni et al. carried out ab initio studies of the atomic structure and the energy barriers for grain boundary sliding in germanium and aluminum. ${ }^{17,18}$ Employing molecular dynamics simulations in conjunction with the embedded atom method (EAM), Chandra and Dang have shown that the coupling between sliding and migration depends strongly on the external conditions that initiate the grain boundary sliding. ${ }^{13}$

In this paper we have studied the interactions of vacancies with the tilt $\Sigma 5$ (210)[001] grain boundary in aluminum using state-of-the-art $a b$ initio total-energy calculations based on the pseudopotential plane wave method. First, we have calculated the vacancy formation energies and atomic structures for a vacancy placed at various positions in the grain boundary region. These results reveal that the grain boundary region may not always be the favorable place to form a vacancy, depending on the location of the vacancy. Second, in order to understand the effect of vacancies on the grain boundary mobility, we have investigated the effect of vacancies on the grain boundary sliding and migration behavior. Aluminum is selected in this study because it is a prototypical simple metal of importance in industrial applications and some of the conclusions we draw from the present study may be extended to other fcc metals as well.

The remainder of this paper is organized as follows: Sec. II describes briefly the computational techniques and the supercell used for the grain boundary calculations. In Sec. III we present the results for the atomic structures and energetics of the pure grain boundary. Section IV contains the results with vacancies introduced at various atomic sites of the grain boundary. The vacancy formation energy at different sites is calculated and compared to that of the bulk system. The atomic relaxation due to the grain boundary expansion and the vacancy-induced contraction will be examined and 
compared. In Sec. V we present the energy profile and the atomic structures for the grain boundary sliding and migration process with and without a vacancy. We find that the vacancy can significantly increase the grain boundary sliding and migration energy barrier, and therefore hinder the grain boundary motion. In order to shed light into the microscopic physics of the combined grain boundary sliding/migration motion, we have examined in detail some critical atomic configurations during the sliding/migration process. In Sec. VI a brief summary and statement of conclusions are presented.

\section{MODEL AND COMPUTATION}

The electronic structure of the grain boundary is calculated by means of the pseudopotential plane wave method based on the density functional theory and the local density approximation. We employed the exchange and correlation potential of Ceperly and Alder as parametrized by Perdew and Zunger ${ }^{19}$ and the norm-conserving nonlocal pseudopotential of Bachelet, Hamann and Schlüter. ${ }^{20}$ The Kohn-Sham wave functions are expanded in plane waves with a kinetic energy cutoff of $14 \mathrm{Ry}$. The atomic structures are considered fully relaxed when the Hellmann-Feynman forces on each atom are smaller than $0.001 \mathrm{Ry} / \mathrm{au}$. For the Brillouin-zone sampling, a $k$-point grid consisting of $(2,7,1)$ divisions along the reciprocal-lattice directions is used according to the Monkhorst-Pack scheme. ${ }^{21}$ Convergence tests have been performed both for the number of $k$-points as well as the number of plane waves. The calculated equilibrium lattice constant $\left(a_{0}\right)$, bulk modulus and cohesive energy are $3.93 \AA$, 80.59 $\mathrm{GPa}$ and $3.26 \mathrm{eV} /$ atom, respectively, in good agreement with the corresponding experimental room-temperature values of $4.05 \AA$, $76.93 \mathrm{GPa}$ and $3.39 \mathrm{eV} / \mathrm{atom}$.

In this work we consider the symmetric $\Sigma 5$ (210)[001] tilt grain boundary in the coincident-site-lattice (CSL) notation. In the CSL notation, the grain boundary is formed through rotation of the two fcc grains about the [001] tilt axis by $36.9^{\circ}$. The boundary is oriented along the (210) plane and the inverse density of CSL sites, $\Sigma=5$, characterizes the unit cell volume of the CSL superlattice. The $\Sigma 5$ [001](210) grain boundary of $\mathrm{Al}$ is simulated by the orthorhombic supercell shown in Fig. 1. The supercell contains 60 atoms distributed in 30 (210) layers parallel to the interface. The dimensions of the supercell along the [120], [001] and [210] directions are $8.78 \AA \times 3.93 \AA \times 26.35 \AA$, respectively. Because of the periodic boundary conditions, the supercell contains two equivalent grain boundaries. However, as will be shown in Sec. III, the separation between the two grain boundaries is large enough to eliminate the interaction between them. For the modeling of vacancies in the grain boundary, a single vacancy is placed at all possible independent lattice sites labeled by an integer $n=0-7$, representing the $n$th layer from the grain boundary plane [the number of independent lattice sites is reduced due to the symmetry between the (001) and (002) planes and that between the two grain boundaries]. It should be pointed out that owing to the periodic boundary condition along [001] axis, we are actually simulating the interactions between a grain boundary

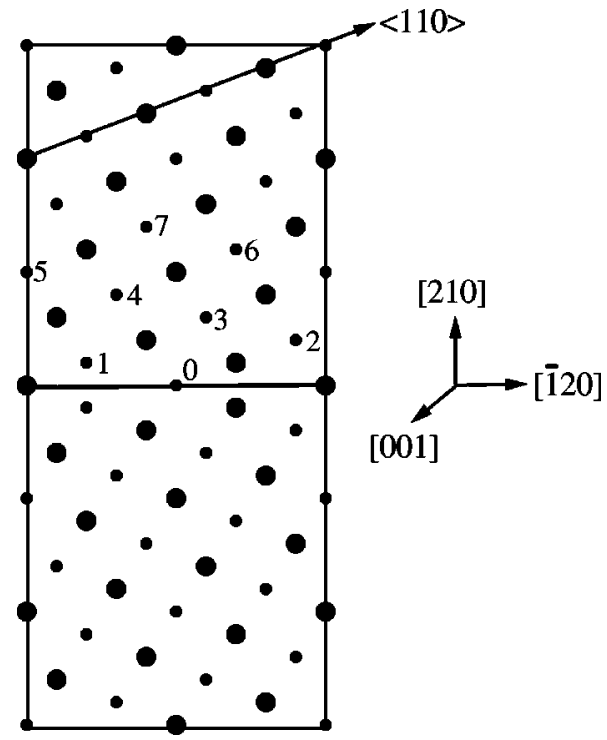

FIG. 1. Supercell for the $\Sigma 5$ (210) [001] tilt grain boundary in $\mathrm{Al}$ viewed along the [001] direction. The large and small circles represent atoms on the (001) and (002) planes, respectively. The integer $(n=0-7)$ represents the $n$th nearest-neighbor layer from the grain boundary interface that a vacancy is placed. The grain boundary plane is indicated by the solid line.

plane and a row of alternating vacancies with separations of $3.93 \AA$. In addition to the grain boundary calculation, we have also computed the vacancy formation energy for $\mathrm{Al}$ bulk by employing a simple cubic supercell with 108 atomic sites to model an isolated vacancy.

\section{PURE GRAIN BOUNDARY}

A pure grain boundary in the following is referred to as the grain boundary without vacancies. The atomic structure of the pure grain boundary before and after relaxation is shown in Fig. 2. The grain boundary volume expansion is clearly seen near the interface and one local measure for such

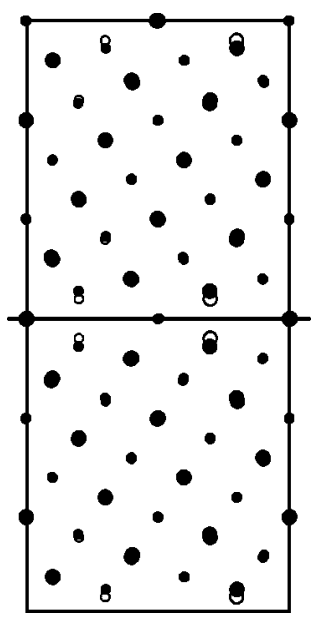

FIG. 2. Unrelaxed (open circles) and relaxed (filled circles) atomic structure for the pure $\Sigma 5$ grain boundary. Large and small circles represent atoms on the (001) and (002) planes, respectively. 


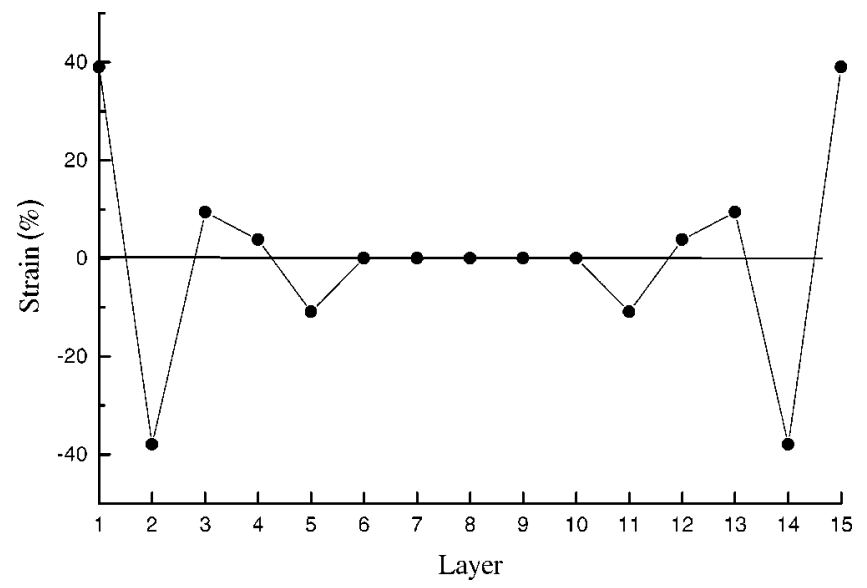

FIG. 3. Interlayer strain (percentage) as a function of the number of layers away from the interface.

an expansion is the relative normal displacement of the two atomic planes closest to the interface. The present calculation yields a local expansion of $0.09 a_{0}$, where $a_{0}$ is the bulk Al lattice constant. This local expansion of $\mathrm{Al}$ atoms in the pure Al $\Sigma 5$ grain boundary is smaller compared to the corresponding expansion of $\mathrm{Al}$ atoms in $\mathrm{Ni}_{3} \mathrm{Al}$ alloy for the same grain boundary. ${ }^{9}$ This is consistent with the fact that there is charge transfer from the $\mathrm{Al}$ atoms to the nearest-neighbor $\mathrm{Ni}$ atoms in $\mathrm{Ni}_{3} \mathrm{Al}$, which in turn reduces the screening charge between the nearest Al-Al ions across the interface, leading to a stronger electrostatic repulsion between the $\mathrm{Al}$ ions in $\mathrm{Ni}_{3} \mathrm{Al}$ grain boundary. In Fig. 3 we present the relative variation (strain) of the interlayer spacing as a function of the layer away from the grain boundary plane. The strain has a symmetric oscillatory profile that reaches its maximum at the first layer and decays into the bulk. The nature of this strain profile can be traced to the Friedel oscillations of the electron distribution which drive the ions to relax in such an oscillatory pattern. ${ }^{22}$ The negligible strain for the layers from 6 to 10 clearly indicates that the atomic relaxation is localized only within 5 layers from the interface. Therefore, the supercell with 15 layers between the two interfaces is large enough to capture the overall properties of the grain boundary.

It is interesting to notice that even after the relaxation, the distance between the nearest-neighbor atoms across the interface is $2.44 \AA$, about $20 \%$ smaller than the corresponding distance of $2.78 \AA$ in the bulk system, showing that a perfect grain boundary may not always have more open space than a bulk. On the other hand, as will be shown in Sec. IV, vacancies can be formed relatively easier in most of the grain boundary region compared to the bulk, giving rise to more empty space in the region. The grain boundary energy can be determined from the difference of the energy of a unit cell containing the grain boundary and a unit cell containing an equal number of atoms in the bulk environment, divided by the grain boundary area. We find a grain boundary energy of $502 \mathrm{~mJ} / \mathrm{m}^{2}$ for the $\Sigma 5$ grain boundary, which is larger than the corresponding EAM value of $351 \mathrm{~mJ} / \mathrm{m}^{2} .^{23}$ This result is consistent with the finding that, overall, the EAM potential

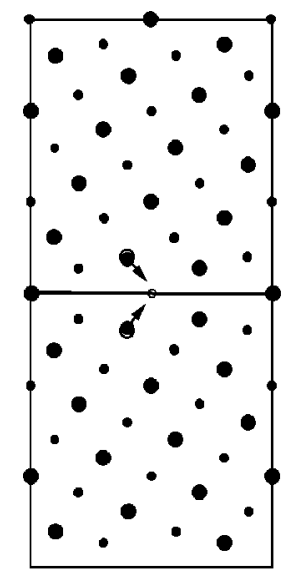

(a)

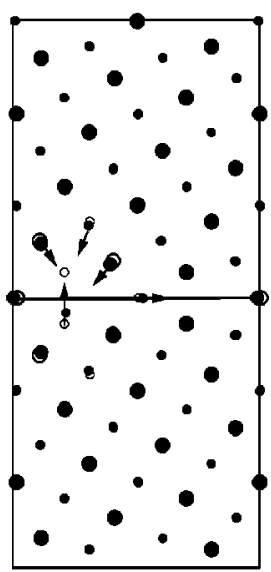

(b)

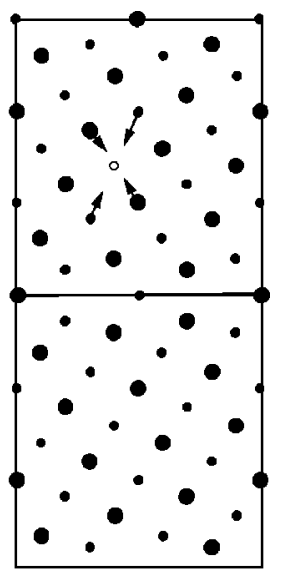

(c)
FIG. 4. Relaxed atomic structures for the grain boundary before (open circles) and after (filled circles) the introduction of a vacancy at the interface (a), the first layer (b), and the seventh layer (c) from the interface, respectively. The large and small circles represent atoms on the (001) and (002) planes, respectively. The arrow shows the direction of the atomic displacement due to the vacancy.

tends to give smaller grain boundary energies compared to ab initio calculations. ${ }^{8}$

\section{GRAIN BOUNDARY WITH A VACANCY}

The relaxed atomic structures of the grain boundary before and after the introduction of a vacancy are shown in Figs. 4(a)-4(c), corresponding to the vacancy placed at the zeroth (interface), first and seventh layers from the interface. In order to gain insight into the interplay between the grain boundary and the vacancies, we have calculated the displacements due to the grain boundary expansion $\left(\mathrm{u}_{G B}\right)$ and that due to the vacancy contraction $\left(\mathrm{u}_{V}\right)$. Both $\mathrm{u}_{G B}$ and $\mathrm{u}_{V}$ are computed for the nearest-neighbor atoms (first shell) from the defects. For the case of the vacancy at the interface [Fig. $4(\mathrm{a})$, although the atomic relaxation is small and localized, the vacancy-induced contraction $\left(\mathrm{u}_{V}=-0.12 \AA\right)$ is much larger than the grain boundary expansion $\left(\mathrm{u}_{G B}=+0.01 \AA\right)$. When the vacancy is placed at the first layer, the atomic relaxation is more dramatic [Fig. 4(b)]. There are seven atomic layers involved in the relaxation triggered by the vacancy and the first shell displacements are $\mathrm{u}_{V}=-0.53 \AA$, while $\mathrm{u}_{G B}=+0.34 \AA$, respectively. In Fig. 4(c) we show the atomic structure with the vacancy placed at the seventh layer (bulk-like). We find $\mathrm{u}_{V}=-0.06 \AA$, which is about $2 \%$ of the nearest-neighbor distance of the bulk lattice and is along the [110] direction. These results are in excellent agreement with the calculations by Turner et al. ${ }^{3}$ for a vacancy in Al bulk both for the magnitude and the direction of $\mathrm{u}_{V}$. In other words, the atomic structure for a vacancy at the seventh layer from the interface completely reproduces the characteristics of a vacancy in the bulk. Along with the fact that the grain boundary expansion $\mathrm{u}_{G B}$ at the seventh layer is zero as discussed earlier, we conclude that the selected supercell for the grain boundary calculations is large enough to yield reliable results. It is also interesting to note that as far as the nearest- 


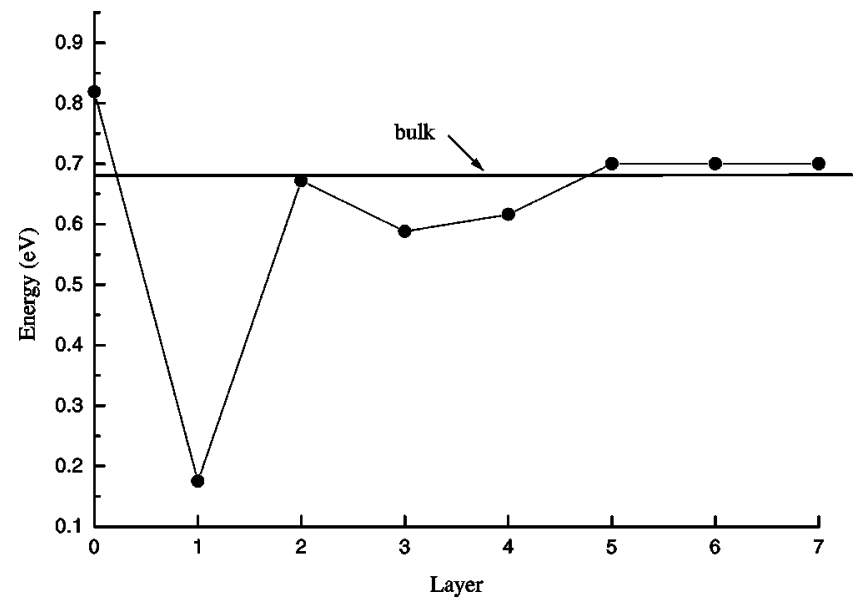

FIG. 5. Vacancy formation energy as a function of the number of the layer away from the interface on which a vacancy is placed. The vacancy formation energy in bulk $\mathrm{Al}$ is denoted by the solid line.

neighbor shell is concerned, the vacancy-induced atomic relaxation is more pronounced than that due to the grain boundary.

In order to clarify whether a grain boundary is always an energy favorable region for vacancies, we have calculated the vacancy formation energy at various layers from the grain boundary plane. The results are presented in Fig. 5, where the straight line denotes the vacancy formation energy in $\mathrm{Al}$ bulk. The striking feature of the energy curve is the large reduction of the vacancy formation energy at the first layer, which strongly suggests the preference of forming a vacancy at the first layer compared to bulk. Although the figure also shows that some other more distant layers in the grain boundary are also energetically favorable for the vacancy formation, one can clearly see that the interface itself is not favorable for forming a vacancy. In fact, there is more than $0.1 \mathrm{eV}$ energy penalty for creating a vacancy at the interface compared to bulk. Note that the vacancy formation energy at the fifth layer from the interface is close to the bulk value, showing the convergence of the energy with respect to the supercell size. In conclusion, the $a b$ initio calculations support the traditional view that grain boundaries act as pathways for vacancy diffusion and that vacancies prefer to segregate to grain boundaries over bulk at low stress. However, we should also point out that one out of five lattice sites in the grain boundary region turns out to be energetically unfavorable for vacancies, namely those sites on the grain boundary plane. It should be pointed out that our results are in consistency with earlier EAM calculations for the same grain boundary in $\mathrm{Cu} .^{12}$

\section{GRAIN BOUNDARY SLIDING AND MIGRATION: EFFECT OF VACANCIES}

In this section we present results for the grain boundary sliding and migration, and the effect of vacancies on the grain boundary motion.

In our $a b$ initio modeling, the grain boundary sliding is simulated quasistatically, namely the top grain is rigidly

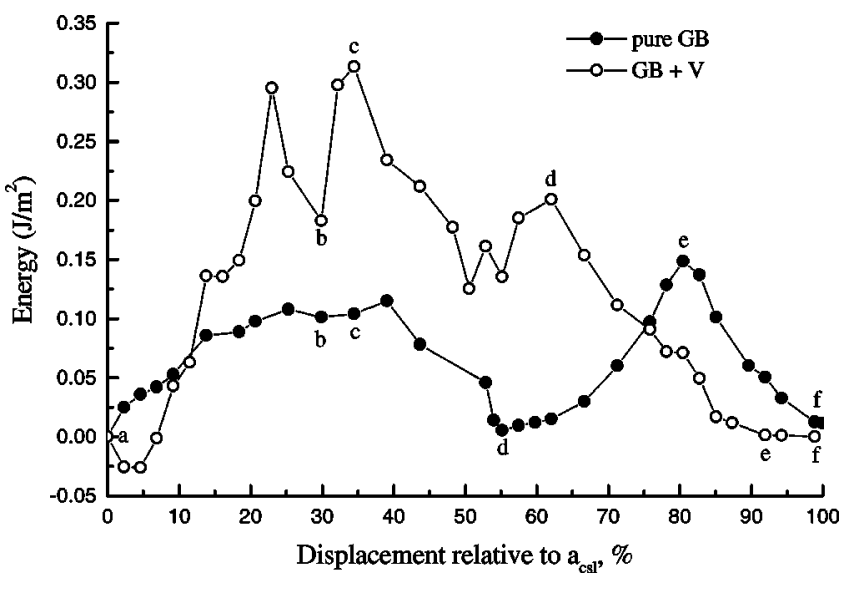

FIG. 6. Relative grain boundary energy as a function of sliding distance expressed as percentage of the lattice parameter, $a_{\mathrm{csl}}$, of the CSL cell along [1 20$]$. The filled and open circles correspond to the energies of the pure grain boundary and the grain boundary with a vacancy at the first layer, respectively. The labels from (a) to (f) represent the special atomic configurations displayed in Figs. 7 and 8.

shifted over the bottom by a series of small specified distances along the interface. The sliding distance is described in percentage of $a_{c s l}$, where $a_{c s l}$ is the lattice parameter of the CSL cell along [120]. Clearly sliding by $a_{c s l}$ will bring the grain boundary back to its original configuration due to the imposed periodic boundary conditions. For each rigid shift, atomic relaxation is performed to locate the closest local energy minimum configuration. In order to understand the effect of vacancies on the grain boundary sliding and migration process, we have calculated the grain boundary energy profile as a function of sliding distance for the pure grain boundary and the grain boundary with a vacancy placed at the first layer from the interface, where it has the lowest vacancy formation energy.

The grain boundary energy profile during the sliding process is shown in Fig. 6, where the filled circles represent the pure grain boundary and the open circles represent the grain boundary with the vacancy, respectively. The labels (a)-(f) on both curves indicate the special atomic configurations which will be explored in detail below. For the pure grain boundary, there are two energy peaks at $30 \%$ and $80 \% a_{c s l}$, the first much broader than the latter; and one energy valley at $55 \% a_{c s l}$. It is important to note that the vacancy triples the energy barrier for sliding and migration, which demonstrates the great hindering effect of the vacancy on the interface mobility. However, the detailed analysis of the atomic structures below shows that the vacancy cannot prevent or even delay the occurrence of the grain boundary migration.

The collective motion of atoms during the grain boundary sliding and migration is essential to understand this seemingly trivial, but in fact complicated process. To shed light on the related physical process, we next examine the atomic configurations associated with the special points (a)-(f) in the energy curves of the pure grain boundary in Fig. 6. The relaxed atomic structures corresponding to (a)-(f) are shown clockwise in Fig. 7. Note that more atoms are displayed in 
(c) $35 \% a_{\text {csl }}$

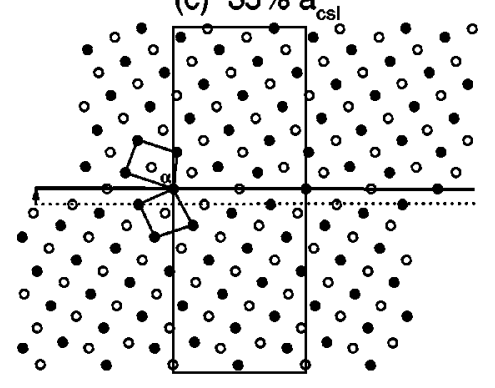

(b) $30 \% \mathrm{a}_{\mathrm{csl}}$

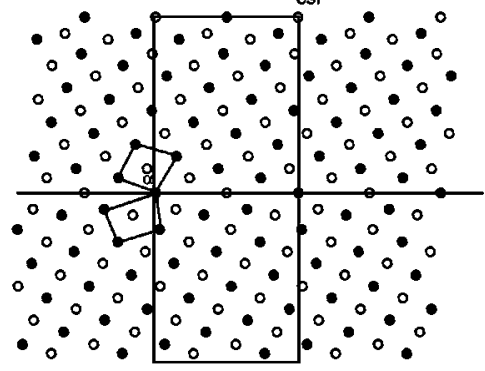

(a) $0 \% a_{\text {css }}$

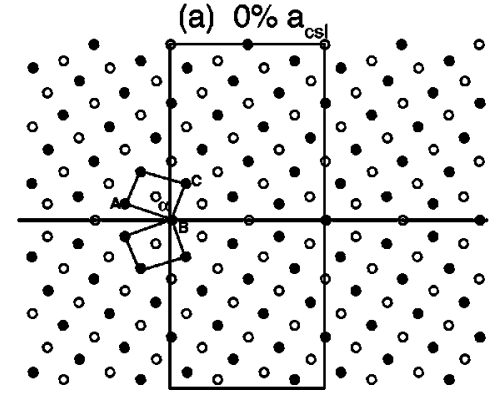

(d) $55 \% \mathrm{a}_{\mathrm{csl}}$

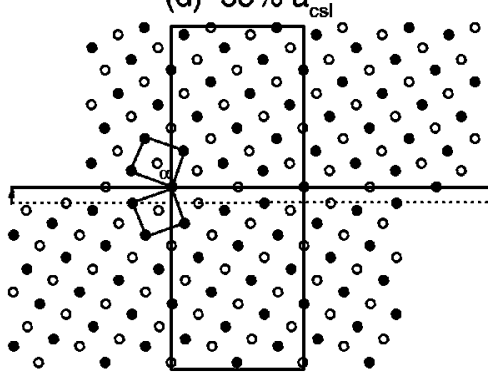

(e) $82 \% a_{c}$
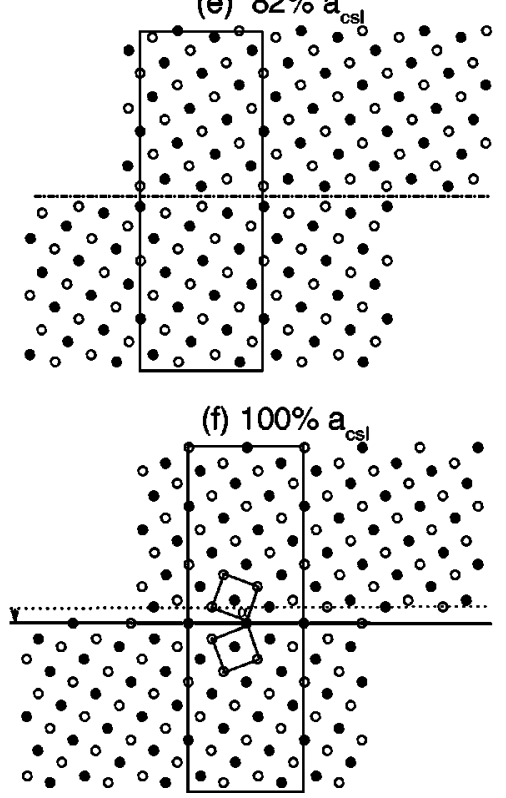

FIG. 7. Evolution of the atomic structure of the pure grain boundary during the sliding process. The snapshots of the grain boundary structures correspond to the special points (a)-(f) on the energy curve for the pure grain boundary in Fig. 6. Filled and open circles represent atoms on the (001) and (002) planes, respectively, and the arrow indicates the direction of grain boundary migration. these figures than the actual atoms used in the supercell, which is depicted by the solid frame. In order to monitor when does grain boundary migration take place, one needs to locate the position of the interface during the sliding process. However, since the grain boundary is not symmetric any more during the sliding process, it is not always obvious to find the new position of the migrating interface. To overcome this difficulty, we propose a simple scheme which allows us to identify the moving interface by following the evolution of the structural unit shown in the figures. The characteristics of this unit is that it consists of two subunits that cross the interface with a common vertex on the interface. These subunits are the two-dimensional unit cells of the distorted fcc lattice. By locating such a unit and the common vertex during the sliding process, one can identify the position of the grain boundary plane. Applying this scheme, we find that the migration of the pure grain boundary starts at $35 \% a_{c s l}$ and ends at $55 \% a_{c s l}$. After the completion of the first migration, the grain boundary has to return to its original position at $100 \% a_{c s l}$ to complete the second migration. For the grain boundary with the vacancy, the migration starts at the exact same sliding distance, $35 \% a_{c s l}$, but ends at about $62 \% a_{c s l}$. The second migration due to the periodic boundary conditions is also observed as in the pure grain boundary.

Figure 7(a) shows the initial atomic structure for the pure grain boundary before sliding. The interface is indicated by a solid line. The angle $\alpha(\angle \mathrm{ABC})$ of the structural unit is slightly distorted from its value of $90^{\circ}$ in the perfect fcc lattice. By examining the various atomic configurations before the first migration $\left(35 \% a_{c s l}\right)$, we find that they are all similar, being different mostly along the sliding direction. More specifically, the angle $\alpha$ of all configurations turns out to be obtuse and one example of such a configuration is shown in Fig. 7(b). When the interface starts migrating, however, the grain boundary experiences an abrupt overall structural transformation, as illustrated in Fig. 7(c). The interface has clearly moved from its original position (dotted line) to the current solid line position, and the angle $\alpha$ has changed from obtuse to acute. This one-layer migration reflects the geometrical necessity for the coupling between migration and sliding. ${ }^{14,16}$ After the onset of the migration, the atomic structures remains akin to each other during the sliding until the migration is fully completed, in which, as shown in Fig. $7(\mathrm{~d})$, the grain boundary recovers its original symmetric structure. Note, that the angle $\alpha$ now changes from an acute angle to a right angle. The dotted and solid lines are the same as in Fig. 7(c). Because of the periodic boundary conditions, the grain boundary has to return to its original position during the remaining sliding process. The critical configuration it has to cross is shown in Fig. 7(e), where the grain boundary experiences the highest energy. The intriguing feature of this configuration is that no boundary plane can be identified, while there is a "mirror" plane (dashed line) across which the atoms on the (001) plane are symmetrically situated to 


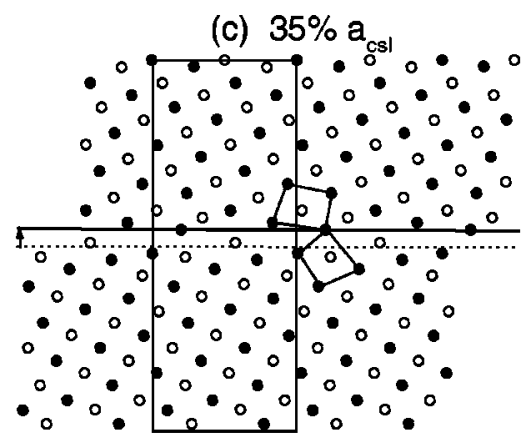

(b) $30 \% \mathrm{a}_{\mathrm{csl}}$

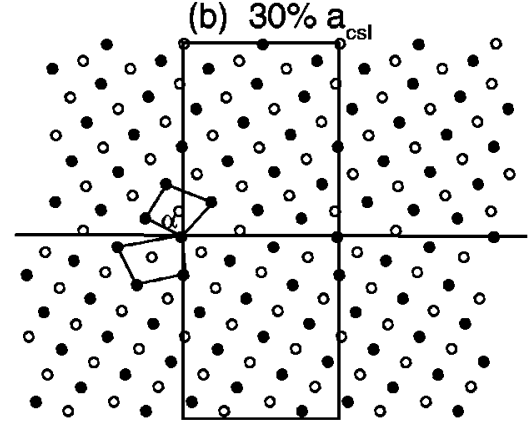

(a) $0 \% a_{c s}$

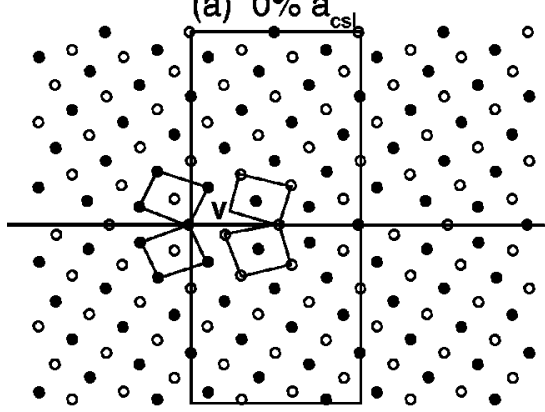

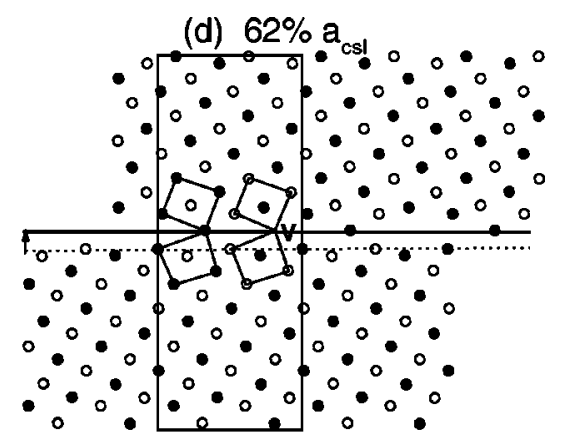

(e) $92 \% a$
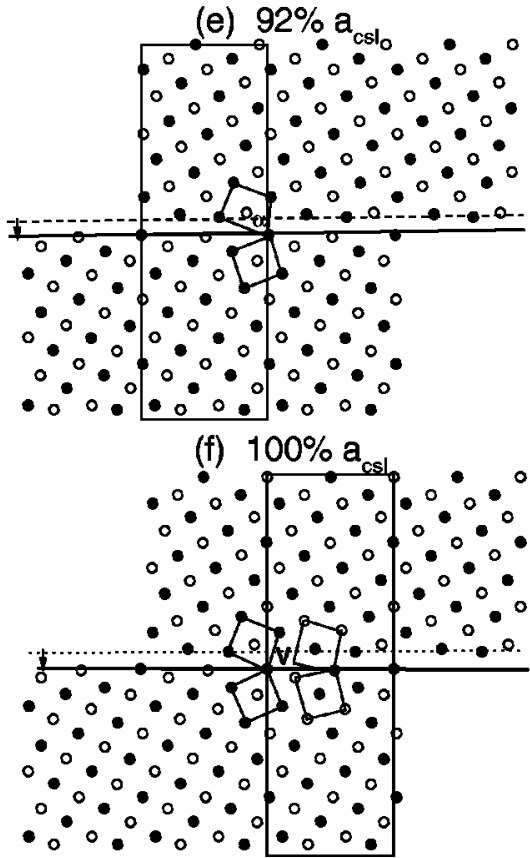

FIG. 8. Evolution of the atomic structure of the grain boundary during the sliding process with a vacancy placed at the first layer above the interface. The snapshots of the grain boundary structures correspond to the special points (a)-(f) on the energy curve for the grain boundary with the vacancy in Fig. 6. Filled and open circles represent atoms on (001) and (002) planes, respectively, and the arrow indicates the direction of grain boundary migration. those on the (002) plane. This half-way plane reflects the transient position of the migrating interface and therefore the energy associated with this configuration gives the energy barrier for the grain boundary migration. Finally, Fig. 7(f) represents the completion of the sliding process where the grain boundary returns to its initial symmetric configuration. The dotted line indicates the position of the interface after the first migration and the solid line shows the current position (also the initial position before the sliding) of the interface.

For the case of the vacancy placed at the first layer from the grain boundary, the sliding energy profile becomes more irregular, with multiple peaks and valleys (Fig. 6). The abundance of the fine grain boundary structures reflects the complexity of the system, so that we shall concentrate only on the most important events. Figure 8(a) shows the atomic structure before sliding and the vacancy is denoted by the letter $\mathrm{V}$. The solid line indicates the current position of the interface. It is found that this initial state no longer corresponds to the lowest energy, which is reached after a sliding displacement of $2 \% a_{c s l}$. There is an energy crossover at $10 \% a_{c s l}$, above which the grain boundary energy with the vacancy becomes larger than the corresponding value of the pure grain boundary, until the next crossover occurs at about $75 \% a_{c s l}$. Although the energy value does not return to zero as in the case of the pure grain boundary when the migration is completed, careful examination of the atomic structures in Fig. 8 indicates the occurrence of the grain boundary migration. Just like the pure grain boundary, the atomic structures remain similar to each other during the sliding process until the first migration takes place at about 35\%. One such configuration is shown in Fig. 8(b), where the angle $\alpha$ is obtuse. The highest energy configuration is reached when the interface migrates one atomic layer upward, shown in Fig. 8(c), in which the dotted and solid lines represent the original and current positions of the interface, respectively. As in the case of the pure grain boundary, the atomic structures at the onset of the migration differ significantly from those before the migration and the atoms near the interface become severely distorted from their positions in the underlying fcc lattice. The grain boundary completes its first migration at about $62 \% a_{c s l}$, where the symmetric structure is recovered [Fig. $8(\mathrm{~d})$ ]. It is important to notice, however, that this symmetric structure with the vacancy (labeled by $\mathrm{V}$ ) at the interface is different from the initial state where the vacancy was at the first layer above the interface. Therefore, the energy difference between the initial state (a) and the current state (d) reflects simply the difference in the vacancy formation energy between the vacancy placed at the interface and the first layer, respectively. This indicates that the vacancy is trapped in the first layer and not able to follow the migrating interface. The grain boundary develops another peculiar configu- 
ration at $92 \% a_{c s l}$, just before the completion of the second migration. A symmetric pattern forms relative to the previous interface (dashed line) and the free volume associated with the vacancy disappears. The off-stoichiometry due to the vacancy, however, is recovered at the symmetry plane (dashed line). The solid line represents the final (also initial) position of the interface during the entire sliding process. The atomic structure of the grain boundary at $100 \% a_{c s l}$ is shown in Fig. 8(f) with the letter V indicating the vacancy. The dotted (solid) lines represent the grain boundary position after the first (second) migration.

\section{CONCLUSION}

We have studied the interaction between vacancies and the $\Sigma 5$ tilt grain boundary in $\mathrm{Al}$, using ab initio pseudopotential calculations, which yield reliable results for both the atomic structure and energetics compared to empirical atomistic approaches. The nearest-neighbor distance across the interface is actually smaller than that in the bulk even after atomic relaxation. We have calculated the grain boundary volume expansion and the vacancy-induced contraction and found that the latter is larger than the former for the nearest- neighbor shell. We have also calculated the vacancy formation energy for a vacancy placed at different layers from the interface. We find that while most of the grain boundary sites have lower vacancy formation energy compared to bulk, those at the interface have higher formation energy. We have investigated the combined grain boundary sliding and migration process with and without a vacancy. We have introduced a simple scheme to identify the migrating interface during the sliding process. We have found that although the vacancy can hinder the grain boundary motion by tripling the energy barrier for sliding and migration, it cannot inhibit or delay the occurrence of migration. In fact, the grain boundary starts the migration at $35 \% a_{c s l}$ regardless if there is a vacancy or not. We have analyzed the atomic configurations during the sliding process in order to gain better insight on this complicated process. Interestingly, the vacancy is found to be trapped at the first layer and not able to follow the migrating interface.

\section{ACKNOWLEDGMENTS}

The research was supported through the US Army Research Office under Grant No. DAAG55-97-1-0093.
${ }^{1}$ Materials Interfaces - Atomic-level Structure and Properties, edited by D. Wolf and S. Yip (Chapman and Hall, London, 1992).

${ }^{2}$ Interfaces in Crystalline Materials, edited by A. P. Sutton and R. W. Balluffi (Oxford University Press, Oxford, 1995).

${ }^{3}$ D.E. Turner, Z.Z. Zhu, C.T. Chan, and K.M. Ho, Phys. Rev. B 55, 13842 (1997).

${ }^{4}$ B. Meyer and M. Fähnle, Phys. Rev. B 59, 6072 (1999).

${ }^{5}$ H. Watanabe and M. Ichikawa, Phys. Rev. B 55, 9699 (1997).

${ }^{6}$ O. Pankratov, H. Huang, T. Diaz de la Rubia, and C. Mailhiot, Phys. Rev. B 56, 13172 (1997).

${ }^{7}$ A. Fazzio, A. Janotti, A.J.R. da Silva, and R. Mota, Phys. Rev. B 61, R2401 (2000).

${ }^{8}$ A.F. Wright and S.R. Atlas, Phys. Rev. B 50, 15248 (1994).

${ }^{9}$ G. Lu, N. Kioussis, R. Wu, and M. Ciftan, Phys. Rev. B 59, 891 (1999).

${ }^{10}$ Y. Mishin and D. Farkas, Philos. Mag. A 78, 29 (1998).

${ }^{11}$ R.G. Hoagland and M.I. Baskes, Scr. Mater. 39, 417 (1998).

${ }^{12}$ M.R. Sorensen, Y. Mishin, and A.F. Voter, Phys. Rev. B 62, 3658 (2000).

\footnotetext{
${ }^{13}$ N. Chandra and P. Dang, J. Mater. Sci. 34, 655 (1999).

${ }^{14}$ G.H. Bishop, R. Harrison, T. Kwok, and S. Yip, J. Appl. Phys. 53, 5596 (1982).

${ }^{15}$ F.R.N. Nabarro and H.L. de Villiers, The Physics of Creep (Taylor \& Francis, London, 1995).

${ }^{16}$ M.F. Ashby, Surf. Sci. 31, 498 (1972).

${ }^{17}$ C. Molteni, G.P. Francis, M.C. Payne, and V. Heine, Phys. Rev. Lett. 76, 1284 (1996).

${ }^{18}$ C. Molteni, N. Marzari, M.C. Payne, and V. Heine, Phys. Rev. Lett. 79, 869 (1997).

${ }^{19}$ J. Perdew and A. Zunger, Phys. Rev. B 23, 5048 (1984).

${ }^{20}$ G.B. Bachelet, D.R. Hamann, and M. Schlüter, Phys. Rev. B 26, 4199 (1982).

${ }^{21}$ H.J. Monkhorst and J.D. Pack, Phys. Rev. B 13, 5188 (1976).

${ }^{22}$ J.-H. Cho, Ismail, Z.Y. Zhang, and E.W. Plummer, Phys. Rev. B 59, 1677 (1999).

${ }^{23}$ S.P. Chen, A.F. Voter, and D.J. Srolovitz, Scr. Metall. 20, 1389 (1986).
} 